\title{
Formation par la recherche et relation éducative en santé
}

\author{
Research training programs and educational process applied to the health sector
}

\author{
Chantal EYMARD'
}

\begin{abstract}
Résumé Contexte : Cette étude sinscrit dans un contexte de "formation par la recherche " des professionnels de l'éducation en santé. But: Identifier la portée d'une "formation par la recherche "selon son degré de référence à un paradigme de pensée, une épistémologie, sur le modèle dominant de l'éducation en santé du professionnel soignant. Matériels et méthodes : Synthèse de la littérature scientifique sur l'épistémologie des connaissances et synopsis de comparaison de six positionnements méthodologiques. Discussion : Les résultats des études en cours mettent en avant qu'en confrontant l'étudiant à des obstacles épistémologiques, une formation par la recherche participe de la modélisation d'un système de pensée, d'une relation aux savoirs savants et aux savoirs expérientiels et donc d'un positionnement dans la relation éducative en santé. Conclusion: Une approche pluri-référentielle de la formation par la recherche, dans une culture du débat épistémologique, est plus formatrice d'une relation éducative fondée sur l'altérité et l'accompagnement des personnes dans la prise de décision, qu'une formation mono-référentielle inscrite dans un modèle mécaniciste et positiviste.
\end{abstract}

Mots clés Epistémologie de la recherche ; formation; relation éducative en santé.

Abstract Background: The study is centred on "research training programs " for professionals in health-care education. Purpose: To identify the impact of a "research training system " in accordance with its degree of reference to a paradigm of thought, an epistemology, on a dominant model of health-care education of professionals in nursing. Material and methods: We have developed a summary of scientific literature on an epistemology of knowledge and a synopsis of comparisons between six methodological approaches. Discussion: The results from this ongoing study emphasize that by confronting students with epistemological obstacles, research training systems help to model a thought system, a relationship to scientific and experiential knowledge, and thus a position in the learning process applied to the health sector. Conclusion: From an epistemological standpoint, an approach using various points of references to research training systems is more likely to create an educational relationship based on otherness and accompanying in decision-making, than a form of training based on a single reference embedded in a mechanistic and positivistic model. Key words Epistemology of research; research training programs; patient education.

Pédagogie Médicale 2006;7:155-64

1- Enseignant-chercheur - Université Aix-Marseille I - Département des sciences de l'éducation - UMR ADEF (Apprentissage, didactique, évaluation et formation).

Correspondance : 29 Avenue Robert Schuman - 13621 Aix en Provence - Cedex 1 - Tél. : 0442571717 - Télécopie : 0442571707 Mailto:chantal.eymard@up.univ-aix.fr 


\section{Introduction}

Quel que soit le champ d'action dans lequel une relation éducative en santé se déroule, elle se construit dans une rencontre singulière entre un professionnel de la santé, caractérisé par ses modèles et ses références, et un sujet qui est lui-même plus ou moins inscrit dans une conception, un modèle de la santé et de l'éducation. La relation éducative s'établit alors dans un rapport conflictuel entre les connaissances scientifiques du moment et des éléments qui font vérité pour le soignant-éducateur comme pour le sujet en éducation. En ce sens, elle se structure dans la connaissance de l'état des lieux des savoirs, la connaissance de l'autre et la connaissance de soi. C'est à partir du débat avec le patient que le soignant peut évaluer la légitimité, la justesse d'une proposition et d'une action et, ainsi, construire une relation respectueuse de l'autonomie du sujet.

Cependant, force est de constater que la pratique éducative en santé est souvent ancrée dans un modèle qui privilégie l'instruction au détriment de l'éducation (OMS, 1998) ${ }^{1}$, voire de l'accompagnement d'un sujet en souffrance $^{2}$. Une pratique plus instructive qu'éducative met l'accent sur l'enseignement des savoirs savants, établis par l'homme de science, au détriment de la vérité du sujet. En œuvrant dans une relation dissymétrique, qui vise implicitement la soumission de l'homme aux normes qui lui sont extérieures (les normes scientifiques), elle est productrice de violence institutionnelle, dans son refus de l'altérité, et renforce les « résistances des patients à tenir compte des messages hétéronomes des soignants $»^{3}$.

Nous postulons qu'il y a un intérêt à repenser la place de la recherche dans la formation des professionnels de santé, au-delà de la seule exigence de former des chercheurs dans une formation à la recherche. Nous pensons nécessaire d'examiner la possibilité d'utiliser la formation par la recherche au service de la construction de compétences professionnelles transposables dans la relation éducative en santé. Plusieurs traits distinguent la formation à la recherche - telle qu'elle est communément mise en œuvre dans le cadre de la formation des professionnels de santéet une formation par la recherche qui serait dédiée au développement des compétences professionnelles en santé (Tableau1). Une formation par la recherche développe des compétences professionnelles en relation éducative, différentes en fonction du caractère monoréférentiel ou pluriréférentiel des méthodes de recherche privilégiées. Les enjeux éducatifs d'une telle option pédagogique, participent du dévoilement d'un dogmatisme plus ou moins masqué dans le champ de la santé ${ }^{3,4}$.
Support du développement de compétences transposables dans les pratiques professionnelles $5,6,7,8$, la formation par la recherche modifie le rapport de l'étudiant au savoir en privilégiant la production de savoir plutôt que la consommation d'un savoir. Former par la recherche nécessite de sortir d'un enseignement "décontextualisé " de la méthodologie ${ }^{8}$, pour privilégier la problématisation d'un objet d'étude, à partir de situations de santé inscrites dans un contexte social, politique et économique. Le développement de l'intelligence critique devient majeur, notamment à travers l'analyse critique de comptes rendus de recherche mais aussi de projets élaborés par les étudiants eux-mêmes.

\section{Professionnalisation des soignants édu- cateurs et formation par la recherche}

La formation des professionnels de santé ne peut pas se satisfaire d'une transmission discursive de savoirs et de savoir-faire ${ }^{9,10}$, ces types d'enseignement se réduisant bien souvent au déploiement du texte du savoir ${ }^{11}$. Ce serait faire fi de l'ensemble des travaux en didactique sur les savoirs-préexistants ${ }^{12,13,14}$ et les obstacles à l'apprentissage ${ }^{15,16}$. Former un professionnel à la relation éducative en santé nécessite un travail sur son rapport aux autres et au monde. Il ne s'agit pas prioritairement d'apprendre à instrumenter son action, mais bien davantage d'apprendre à faire avec l'incertitude dans le travail continu à la connaissance de soi et à l'écoute de l'autre ${ }^{17}$. Développer cette compétence ne peut donc pas s'abstraire d'une formation au questionnement éthique.

Un éducateur en santé qui est surtout préoccupé de la toute-puissance du savoir savant et de l'objectivation par la preuve privilégie une relation éducative basée sur l'instruction, l'ajustage physique, psychologique et culturel, la maitrise de la santé des sujets et des groupes. Les limites d'un tel modèle sont décrites par de nombreux auteurs ${ }^{18-23}$. Il est peu performant en matière de résultats, notamment en ce qui concerne l'augmentation des complications dans les maladies chroniques et des conduites addictives. Il soumet le patient à la violence de l'expert en santé, dans un assujettissement à la norme scientifique. Le soignant, dans la toute-puissance de son désir de convertir le sujet à l'adoption de règles de "bonne santé ", nie sa singularité et son désir.

Pour sortir d'une position dogmatique en santé et concevoir une relation éducative située davantage dans l'accompagnement d'un sujet que dans l'instruction, il est néces- 
Tableau 1 :

Comparaison de la formation à la recherche et par la recherche

Formation à la recherche

Transmission de savoirs sur :

- la méthodologie de la recherche et l'épistémologie des connaissances

- les outils et techniques de recueil et de traitement des données

Enseignement décontextualisé des pratiques professionnelles en santé

\section{Formation par la recherche}

saire d'initier les professionnels de santé au « lâcher prise " sur leurs certitudes. Si l'apprentissage à être en relation éducative s'établit sur des savoirs en santé, il mobilise un " savoir analyser "sa pratique. Réfléchir sa pratique ${ }^{24,25}$ questionne les valeurs, les croyances, les certitudes, les modèles "de relation à l'autre ", "d'être au monde ", l'identité du sujet.

L'expérience de la relation éducative en santé confronte le sujet apprenant à ses certitudes, ses tendances en situation. La clinique est propice à l'élaboration de savoirs nouveaux dans la mise en rapport de l'expérience aux savoirs préexistants. Certaines formations privilégient des dispositifs d'apprentissage par problèmes qui invitent à élaborer des savoirs pour les surmonter. En confrontant l'étudiant à des obstacles épistémologiques, former par la recherche participe à la modélisation d'un système de pensée, d'une relation aux savoirs savants et aux savoirs expérientiels et donc du développement d'un certain positionnement dans la relation éducative. Il ne s'agit pas, pour autant, de nier l'influence des modèles et savoirs en santé, des modèles de l'apprentissage et de l'évaluation véhiculée par la formation.

\section{Des compétences en recherche aux capacités et babiletés mises en cuvre dans la relation éducative en santé}

Nous convenons avec Perrenoud ${ }^{26}$ que former par la recherche peut :
Développement de compétences transposables dans les pratiques professionnelles :

- production de savoirs par l'étudiant

- motivation et appropriation du savoir par une pédagogie du projet ${ }^{4}$

- Enseignement contextualisé

Problématisation de l'objet d'étude à partir de situations de santé inscrites dans un contexte social...
- favoriser « un découpage plus analytique et plus fin de la réalité »;

- développer une tendance "à mieux voir le caché, le refoulé, le non dit ";

- inciter « à mieux prendre en compte la différence et la diversité";

- modéliser « une forme de va-et-vient entre théorie et pratique, d'articulation entre pensée et réalité »;

- initier à l'auto-questionnement ${ }^{27}$ et à l'analyse de ses expériences ;

- contribuer au « lâcher prise " sur les certitudes, au développement de la curiosité, au questionnement permanent des pratiques et des savoirs savants ;

- élucider des phénomènes, des situations.

Que ce soit dans le cadre des formations cliniques ou dans celui des formations à la recherche, la référence épistémologique est souvent univoque. En s'inscrivant dans une perspective positiviste, elle transmet une façon de considérer le rapport au savoir. A partir d'une formalisation des capacités et habiletée ${ }^{28}$ sous-jacentes aux compétences clés visées dans une formation par la recherche (problématisation, méthodologie), nous avons identifié les capacités et habiletés transposables dans une relation éducative en santé. Le soignant-éducateur compétent mobilise des savoirs et des ressources personnelles et les organise en fonction de la singularité de chaque situation. Il n'est donc point possible de définir a priori des standards à observer. Par contre, certaines tendances peuvent se décrire et ainsi offrir des repères pour une analyse des pratiques. 


\section{Apprentissage à la problématisation et développement de compétences en rela- tion éducative en santé}

Dérivé de pro-ballien (pro: devant, ballein : jeter), la notion de problème indique tant la question à résoudre que la controverse, le débat, la discussion, autour d'un phénomène. En s'appuyant sur la pensée d'Aristote, Falcy différencie la question du problème. "Un problème est un nœud, un embarras, une impasse, une aporie. La question est trop tendre, trop attractive de réponses pour être un problème. Un problème fait barrage, alors qu'une question fait pont ${ }^{29}$. Inscrit dans la pensée dialectique, "le problème est, d'une part de nature paradoxale, en allant à l'encontre de l'opinion commune et d'autre part, il est le lieu d'une contradiction ${ }^{29}$. Le travail de problématisation n'est pas réservé au chercheur. La réflexivité d'un agir professionnel en relation éducative en santé, s'appuie sur des compétences à problématiser des situations de santé. Le soignantéducateur est confronté à des situations-problèmes, qu'il cherche à comprendre dans toute leur complexité et leur singularité. Il problématise la situation de santé, dans un rapport dialectique entre l'avancement des savoirs en santé et les savoirs construits dans l'expérience et les choix de vie du patient. En ce sens, toute relation éducative en santé est créative et innovante.

Le développement de compétences en problématisation ne peut pas se suffire d'un enseignement des opérations à réaliser. Chaque situation de formation est une occasion à saisir pour développer cette compétence, à condition que les situations problèmes ne soient pas transformées en questions-réponses par le formateur. Nous comprenons alors qu'il est difficile d'initier cette compétence en s'inscrivant uniquement dans la rationalité expérimentale et dans celle des savoirs établis comme vérité (s) scientifique (s). La confrontation à un problème sollicite les capacités et habiletés du formateur pour susciter la créativité et l'inventivité des étudiants. La formation par la recherche est alors une occasion à saisir pour accompagner l'étudiant en situation concrète et l'aider à :

- contextualiser un objet d'étude d'un point de vue historique, économique, politique et social ;

- définir l'état des lieux des savoirs sur la question;

- réguler ses orientations de recherche en confrontant

l'avancement des savoirs aux pratiques ;

- centrer ou décentrer l'objet ;

- construire un cadre de lecture théorique en travaillant les liens de convergences ou de contradictions entre les différents concepts, modèles et les théories ;

- dialectiser sa pensée ;

- s'initier à la créativité.

A partir de ces capacités développées par l'apprentissage à la problématisation d'un objet de recherche, nous avons identifié les capacités et habiletés visées qui peuvent être transposables dans une relation éducative en santé ; elles sont présentées dans le tableau 2.

L'accompagnement d'un apprenti-chercheur dans la problématisation d'un phénomène ou d'une situation est une étape essentielle du processus de recherche. Un phénomène n'est pas une réalité objective, il désigne ce qui est perçu par un sujet à travers l'ensemble de ses sens. Notre sensibilité et notre entendement font partie du monde des phénomènes. L'objectivité renvoie à une vérité de l'objet et tend à exclure la subjectivité de tout être humain, qui pourtant est à l'œuvre dans un processus de recherche. Les étudiants qui sont inscrits dans une culture de la science comme vérité objective, de la technique en tant que procédure opératoire au service de la question à résoudre plutôt qu'à débattre, et des réponses apocritiques ${ }^{30}$ qui ferment le débat, sont peu enclins à dialectiser leur pensée. En développant des capacités à problématiser, le soignantéducateur s'initie à la dialectique et à l'approche complexe des situations de santé.

Dialectiser développe le processus dialogique de questionnement et met l'accent sur l'impossibilité d'apporter une réponse unique au problème. La tendance à être dans la résolution pour l'autre et les a priori de l'éducateur sont bousculés, ouvrant alors un espace au différent. "Problématiser pour être capable de débusquer les "fausses solutions" que nous dictent en toute logique nos préjugés non identifiés et/ou nos analyses trop simplistes ${ }^{31}$ devient dès lors une approche alternative. En clarifiant les notions d'usage, en questionnant les affirmations, les lois, les vérités scientifiques, l'apprenti-chercheur est confronté à ses propres préjugés. L'entraînement à cette capacité pourrait développer une conception de l'éducation en santé dans laquelle la contradiction est inclusive de la relation qu'il construit avec le patient. On peut imaginer alors que le soignant-éducateur s'ouvre aux comportements et arguments contradictoires chez le patient. En mettant en exergue la capacité à faire des allers-retours entre le concret et l'abstrait, la problématisation réactive la confrontation entre le savoir académique du moment, son origine et sa destination, en d'autres termes, entre savoir et sens des connaissances. Elle instruit autant à l'actualisation des savoirs en matière de santé, qu'à la mise en tension des 


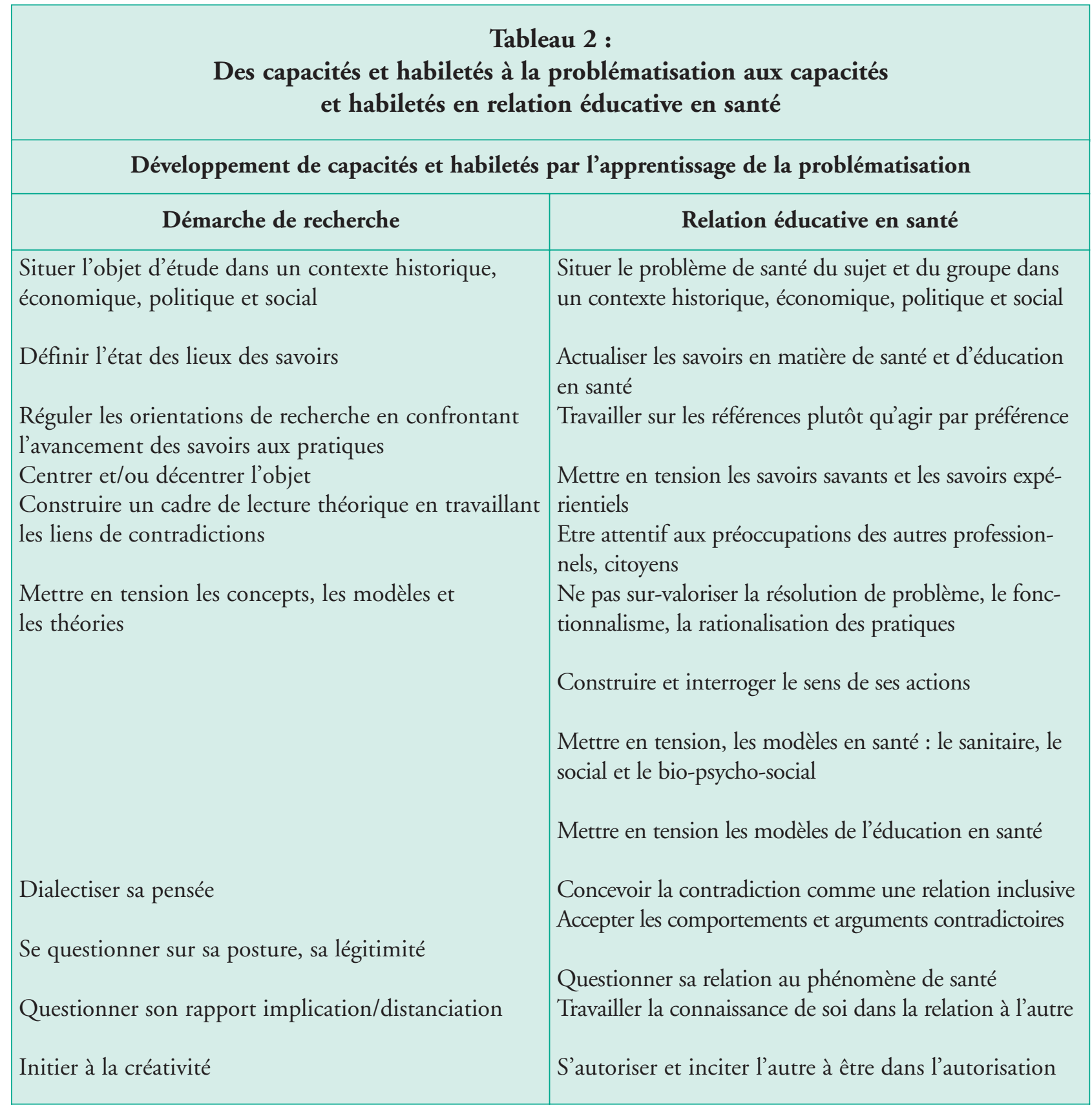

savoirs académiques avec les savoirs d'expériences. En questionnant la valeur épistémologique des savoirs d'actions et des pratiques professionnelles, l'étudiant est invité à " penser sa pratique " ${ }^{32}$. Problématiser réclame écoute et observation, conformation et confrontation, mais aussi acceptation et refus des apparences, des évidences, reconnaissance et métissage de points de vue variés. «Toute problématique est une tension entre l'en-soi et le pour-soi » ${ }^{33 .}$
La recherche en santé travaille des objets qui ne sont pas dénués de sens et confrontent le chercheur à sa subjectivité. L'apprenti chercheur, dans son désir de connaître, se confronte à l'absence de neutralité vis-à-vis du phénomène qu'il souhaite étudier. En œuvrant pour la connaissance de soi dans son rapport à un objet de recherche, mais aussi dans son rapport au monde et à la connaissance, l'étudiant cultive une relation éducative réflexive. En construisant du sens pour lui et pour la communauté (scientifique et profes- 
sionnelle), il développe sa capacité à s'autoriser à être dans la relation, ce qui invite le patient à être lui-même dans l'autorisation. Exigeante de références et motrice de l'innovation et de la créativité, la problématisation participe d'une relation éducative en santé plus créative que standardisée, conçue comme une œuvre qui s'élabore dans l'écoute de la singularité du sujet.

Construire une problématique s'appuie aussi sur un « savoir argumenter ", sur un "savoir prendre position ». Le soignant-éducateur, à travers cet exercice, est amenéà problématiser " ses appartenances, ses repères, ses territorialités » ${ }^{34}$, et à développer sa personnalité professionnelle.

\section{Du choix d'une méthode de recherche à une approche de la relation éducative en santé}

Le débat épistémologique a permis de dépasser le caractère absolu d'une vérité par rapport à deux grandes traditions scientifiques (positivisme et herméneutique) et celui d'une méthode unique pour valider les faits sociaux. Quelle que soit la méthode empruntée, aucune recherche ne peut saisir le phénomène étudié dans sa totalité, encore moins en sciences de la santé où l'éthique ne permet pas de réduire le sujet à l'état d'objet aux contours nettement délimités. Il est nécessaire d'assumer le fait de travailler avec un point de vue particulier, qui permet de regarder seulement une partie de la réalité. "Chaque méthode "invente" son objet de recherche et le traite ensuite comme un Tout, alors qu'il ne s'agit que d'un fragment ${ }^{34}$. L'objet de recherche ne préexiste pas en tant que tel, il se construit dans le regard du chercheur, l'attention qu'il lui porte, la manière dont il l'aborde et l'intentionnalité qui conduit son projet de connaissance ${ }^{35}$. "Quand il s'agit explicitement d'objets, tout à l'opposé, reconnus simultanément en tant que sujets, auteurs (et non seulement acteurs), porteurs de projets et, de ce fait, inscrits dans une temporalité-durée, au regard d'une visée de production de connaissances nouvelles, toutefois finalisée à partir d'un souci praxéologique (optimisation de l'action, aide à la décision), l'ordre du témoignage prend délibérément le pas sur celui de la preuve; en d'autres termes, l'expérience de l'affecté (Jeanne Favret-Saada), celle de l'éprouvé, plus encore que celle de la preuve, vont revêtir une réelle consistance dans le cadre de ces formes de recherches qui iront jusqu'à prendre à la limite pour objet le travail d'exister $»^{36}$. Il ne s'agit pas, pour autant, d'exclure une méthode, un paradigme scientifique pour se fondre dans un autre qui serait considéré comme meilleur. A l'écoute de la pluralité des mondes ${ }^{37}$, la recherche en santé s'ouvre à un regard pluriel des phénomènes qu'elle étudie.

Le choix d'une méthode de recherche participe grandement de la construction de l'objet mais aussi de la posture du chercheur. La recherche n'est pas « indépendante des phénomènes qu'elle étudie, d'un double point de vue : d'une part son introduction, son déroulement aussi bien que ses résultats ne sont pas sans incidence sur les dits phénomènes; d'autre part les perspectives épistémologiques actuelles [...] nous rappellent que le chercheur n'est pas extérieur au monde qu'il étudie ${ }^{35}$. Si le choix d'une méthode de recherche peut relever d'un a priori épistémologique, il peut aussi se construire à partir d'un débat sur l'intérêt et les limites de différentes méthodes au regard de l'état des lieux des savoirs et de la relation aux acteurs de la recherche. En incitant les étudiants à travailler sur plusieurs options méthodologiques, la formation conduit à appréhender l'élaboration de savoirs académiques comme " une aventure incertaine qui comporte en elle-même, et en permanence, le risque d'illusion et d'erreur ${ }^{38}$. Cette initiation au débat épistémologique confronte les tendances à formuler des énoncés de manière dogmatique et participe ainsi au processus de professionnalisation.

Nous avons réalisé, avec l'aide d'un groupe d'experts en méthodologie de la recherche en sciences humaines, un synopsis de comparaison de cinq positionnements méthodologiques dans une intervention de recherche (expérimental, différentiel, clinique, ethnologique, systémique), à partir de cinq critères majeurs (origine, postulats, posture du chercheur, caractéristiques de la question de recherche, passages obligés pour la construction des savoirs scientifiques) ${ }^{39}$. L'avancement de notre réflexion nous convie à dire aujourd'hui que les limites épistémologiques ne peuvent pas se réduire dans une combinaison, ni une juxtaposition des différentes méthodes. Si chaque méthode de recherche est restrictive pour l'étude d'un phénomène, elle en propose une approche particulière.

- Si la science expérimentale a contribué à formaliser et instrumenter la recherche en santé, elle l'a aussi engluée dans les principes du positivisme et du mécanicisme ${ }^{21}$. En s'opposant au monde des croyances et de l'obscurantisme, elle a produit un discours objectivé et raisonné sur les phénomènes sociaux déclarés observables et constitués par les acteurs eux-mêmes. Ses préceptes ont dirigé l'approche de la construction des savoirs et de la connaissance dans une épistémê de la généralisation, de la systématisation de la notion de loi, de la maîtrise d'un réel, de la réduction au simple, au visible, au compréhensible, et de l'observation systématique des faits ${ }^{40}$. Lorsque la recher- 
che en santé fait exclusivement référence à la rationalité expérimentale, elle prône la maîtrise des choses et des phénomènes. L'affirmation que seule la science expérimentale est valide pour la connaissance renforce le poids de la démarche de la preuve sur celle de l'élucidation et attribue au savoir construit le statut de vérité scientifique, ne laissant que peu de place pour la vérité du sujet ${ }^{41}$. La production de savoirs objectivés met en péril la singularité inhérente à la condition de sujet, en le réduisant à un objet de conformation sociale, à un standard. Cette indifférenciation du sujet et de l'objet, se concrétise dans certaines pratiques éducatives en santé qui mettent l'accent sur la normalisation des conduites et des attitudes, la standardisation des réponses apportées, des tests et des traitements, et sur la transmission de savoirs objectivés au détriment d'une éducation à l'exercice du singulier dans le social.

- En méthode différentielle, le chercheur est centré sur les variations de réponses des sujets par rapport à un phénomène extérieur. Il identifie les caractéristiques individuelles ou groupales, facteurs a priori signifiants de la variabilité des comportements, des pratiques, ainsi que l'existence ou non de liens entre ces dimensions. Les phénomènes sont observés dans un environnement, en tentant de prendre en compte un ensemble d'interactions. Le jugement de corrélation prend le pas sur le causalisme. La variabilité des comportements « n'est plus abordée comme l'expression d'une erreur ou d'une imperfection de nos mesures, non plus comme une entrave à une description de l'homme universel ou de processus universels. Elle est abordée au contraire pour elle-même, comme une propriété essentielle du fonctionnement psychologique individuel et de la diversification des fonctionnements psychologiques au sein d'une population $»^{42}$.

- En tant que méthode de recherche, la clinique du sujet ou psychophénoménologie participe à l'élaboration de savoirs académiques à partir de la vérité du sujet (non réduit de manière dogmatique à un objet), de sa parole en tant que travail d'une énonciation et d'une interprétation. Elle peut passer à côté de sa spécificité, lorsqu'elle tente d'acquérir un statut scientifique dans un modèle où l'objectivation gomme toute subjectivation, d'où notamment l'intérêt de différencier recherche clinique de méthode clinique, la notion de recherche clinique étant réservée alors à l'ensemble des recherches réalisées auprès du patient, quelle que soit la méthode de utilisée ${ }^{43}$. Lorsqu'un chercheur privilégie une méthode clinique ou une approche psychophénoménologique $^{44}$, les structurations de l'environnement que le sujet opère par projection de significations sont forte- ment considérées ${ }^{33}$. La parole du sujet est l'expression de sa vérité. L'interprétation n'est pas " une application d'un savoir extérieur, mais un savoir qui surgit de cette relation et de sa temporalité. Il ne s'agit pas d'une explication, ou de la recherche d'une prise de conscience, mais d'un "acte" qui fait sens dans un contexte donné » ${ }^{45}$.

- Dans les méthodes de l'ethnologie, l'ethnographie et de l'anthropologie, les faits sociaux ne sont pas considérés comme des objets stables qui conduiraient à une réalité objective et généralisable. Ils sont une réalité en euxmêmes "en tant qu'accomplissement continu des activités concertées de la vie quotidienne des membres, qui utilisent, en les considérant comme connus et allant de soi, des procédés ordinaires et ingénieux pour cet accomplissement $"{ }^{46}$. L'intervention de recherche est construite en opposition à la pensée déductive classique, au déterminisme, à la conformisation de l'Homme à des faits prédictifs, inéluctables et généralisables. Le chercheur est un acteur impliqué dans le groupe étudié. Le réel y est considéré comme un construit par des acteurs dans un groupe auto-marqué.

- Considérant l'absence de linéarité des phénomènes, l'intervention de recherche construite à partir d'une méthodologie systémique récuse l'exclusivité des relations de cause à effet et propose de composer avec la complexité des phénomènes sans les isoler de leur environnement. Elle œuvre pour la construction de savoirs pratiques à partir de la modélisation du fonctionnement d'une organisation éducative. Le chercheur intervient dans le système. Il s'inscrit dans une logique d'invention et non de généralisation. L'interaction objet/sujet est constitutive de la connaissance. Le modèle " laisse plutôt émerger les enchevêtrements des interventions sociales et leurs interrelations ${ }^{47}$. Les connaissances sont validées avec les acteurs du système. Praticiens et chercheurs sont co-auteurs de la recherche.

Chaque méthode de recherche, définissant une manière de construire des savoirs, s'inscrit dans un paradigme témoin d'une vision du monde, de l'humain, de la santé et de l'éducation. Certes, au-delà de la méthode, il y a l'apprenti-chercheur qui s'y réfere et l'enseignant qui accompagne les travaux de recherche. Nous pouvons cependant penser qu'une formation par la recherche inscrite de façon prédominante dans le paradigme mécaniciste et positiviste aura tendance à accentuer les propensions de normalisation des professionnels de santé au nom de la "bienfaisance pour l'autre». Une telle formation œuvre pour une relation éducative basée sur l'instruction, la transmission des savoirs objectivés par la science et le 
changement de comportement des personnes et des groupes sociaux vers l'adoption des normes en vigueur. Une approche pluri-référentielle de la formation par la recherche, dans une culture au débat épistémologique, sera plus formatrice d'une relation éducative fondée sur l'altérité et l'accompagnement des personnes dans la décision qu'une formation mono-référentielle inscrite dans un modèle mécaniciste et positiviste.

Une dominante de formation par la recherche inscrite dans le paradigme de l'herméneutique, de la phénoménologie aura tendance à développer les compétences des professionnels de santé à inscrire la relation éducative dans la rencontre avec un « sujet désirant » et « la capacité à faire émerger de l'altérité dans une relation de parité asymétrique $"$. C'est bien dans cette considération du processus d'altérité, à partir du travail sur la connaissance de soi et de ses implications dans un agir professionnel d'éducation en santé des individus et des groupes, que la différence de l'autre peut être entendue. Nous pourrons alors imaginer une éducation en santé inscrite dans la réalité subjective du patient ou des groupes sociaux considérés, où les savoirs d'expérience ne sont pas entendus uniquement comme des obstacles à l'apprentissage, ni comme des erreurs de représentation à corriger, mais comme de réels savoirs qui peuvent produire la régulation des savoirs savants.

Sans nier l'intérêt de la recherche expérimentale, ni celui de la méthode différentielle, nous proposons aux formations des professionnels de santé de sortir d'une approche mono-référentielle de la recherche et de la survalorisation du paradigme mécaniciste et positiviste. Il s'agit de dépasser le causalisme et le déterminisme en santé, inscrit dans l'imaginaire de la toute-puissance et de la maîtrise des hommes sur le monde, et de mettre l'accent sur l'autonomie du sujet ou des groupes sociaux. La personne en éducation peut alors être considérée comme un sujet dans sa capacité à décider par lui-même de ses choix, en tenant compte de l'environnement dans lequel il vit. Le soignant accompagne le patient dans une prise en charge active de sa maladie, de ses soins et de sa surveillance, tout en étant en éveil sur les limites d'un discours sanitaire complètement basé sur l'autonomie et la responsabilité du patient ${ }^{2}$.

\section{Conclusion}

Nous soutenons qu'une formation par la recherche développe des compétences en relation éducative, différentes selon que les méthodes de recherche privilégiées s'inscrivent dans une approche mono-référentielle ou pluri- référentielle. Former les professionnels de la santé, dans une approche pluri-référentielle des méthodes de recherche les initie au débat épistémologique. Le double processus de subjectivation des savoirs et d'objectivation des relations au savoir accompagne le sujet en apprentissage dans l'élaboration d'un personnage professionnel aux prises avec des différences culturelles, des différents mondes des patients.

Au patient objet d'une relation éducative instaurée et dirigée par le professionnel, peut se substituer un patient sujet, co-auteur, co-acteur et décideur dans la relation éducative en santé. La relation éducative se construit alors dans le débat critique entre les patients et les professionnels de la santé, chacuns aux prises des savoirs savants, des énoncés scientifiques et de ses expériences en santé. Ainsi conçue, elle ouvre un espace de décision pour le patient en fonction de chaque situation de santé. "L'un des paradoxes du discours soignant, empreint des notions d'autonomie et de responsabilité, est d'imposer simultanément une norme médicale intransigeante (type objectif glycémique, ration calorique quotidienne, plan d'action dans l'asthme, par exemple...) alors qu'en toute logique, le plein exercice par le patient de son autonomie et de sa responsabilité devrait l'amener à proposer ses propres normes, de telle manière qu'une négociation s'engage ${ }^{10}$.

Dans une telle conception de la relation éducative, l'écart à la norme et aux savoirs académiques change de statut. Il n'est plus obligatoirement erreur. L'erreur n'est plus considérée comme faute mais prétexte à questionner la logique, la genèse du raisonnement. Si l'erreur est déplaisante, voire objet de souffrance pour la plupart des sujets, «c'est peut-être parce que notre société est rentrée globalement depuis un siècle ou deux dans une dynamique de changement mais que, faute d'avoir perçu la rupture épistémologique produite par la signification que l'erreur prend dans la démarche scientifique ou dans l'apprentissage, les éducateurs et les enseignants continuent, à leur insu, à être les vecteurs d'une représentation de l'erreur d'une époque où, pour des raisons sociales, il fallait inhiber les individus dans leur pulsion d'exploration $"^{49}$. L'éducation en santé peut se concevoir dans une culture du débat ${ }^{50}$, de la confrontation et du dissensus, dans laquelle les savoirs que les patients élaborent en vivant au quotidien leurs problèmes de santé sont reconnus. La manière dont les savoirs d'expérience se constituent, ainsi que la nature même de ses savoirs sont sources de régulation de l'éducation en santé des patients. 


\section{Références}

1. OMS. Education thérapeutique du patient. (1998). In : Bureau régional pour l'Europe, Copenhague, Recommandation d'un groupe de travail de l'OMS, version française, septembre 1999.

2. Deccache A, Lavendhome E. Information et éducation du patient, des fondements aux méthodes. Bruxelles: De Boeck, 1989.

3. Lecorps P, Paturet J-B. Santé publique, du biopouvoir à la démocratie. Rennes : ENSP, 1999.

4. Gadamer H-G. Philosophie de la santé. Paris : Grasset, 1993.

5. Altet M, Fabre M. Logiques et problématiques d'articulation formation/recherche dans les dispositifs de professionnalisation. Recherche et formation 1994;17:77-92.

6. Lemery B. Usages de la recherche en situations de formation professionnelle : le point de vue d'un enseignant-chercheur. Recherche et formation 1994;17:921 .

7. Demailly L, Zay D. Gestion de la recherche et formation de formateurs d'enseignants. A quelles conditions institutionnelles la recherche peut-elle être un outil de formation pour un groupe professionnel? Recherche et formation 1994;17:47-63.

8. Van der Maren J-M, Blais J-G. Quelle recherche et avec quel rôle dans la formation professionnelle en éducation? Recherche et formation 1994;17:35-41.

9. Ardoino J. De l'accompagnement, en tant que paradigme à René Lourau. Pratiques de Formation/Analyses 2000;40:5-19.

10. Ivernois (d') J-F, Gagnayre R. Apprendre à éduquer le patient. Approche pédagogique. Paris : Maloine, (1995) 2004.

11. Chevallard Y. La transposition didactique. Du savoir savant au savoir enseigné. Grenoble : La Pensée Sauvage, 1991.

12. Johsua S, Dupin J-J. Introduction à la didactique des sciences et des mathématiques. Paris : PUF, 1993.

13. Sensevy G. Institutions didactiques. Paris : PUF, 1998.
14. Ravestein J. Autonomie de l'élève et régulation du système didactique. Bruxelles : De Boeck, 1999.

15. Bachelard $G$. La formation de l'esprit scientifique. Paris : Vrin (1938), 1996.

16. Brousseau G. Théorie des situations didactiques. Grenoble: La pensée Sauvage, 1998.

17. Vial M. Les sciences de l'éducation et les personnels de santé, une histoire prometteuse. Soins cadres 2003;45:29-33.

18. Bury J. Education pour la santé Conceptions, enjeux, planifications. Bruxelles : De Boeck, 1988.

19. Gadamer H-G. Le Banquet Des Philosophes. Paris : Grasset, 1998.

20. Lecorps Ph. La santé vécue par le sujet. Évaluer l'éducation pour la santé, concepts et méthodes. Bordeaux : INPES, Séminaires Université Bordeaux, 1998:19-27.

21. Jouquan J. L'évaluation des pratiques confrontées à l'exigence de la preuve scientifique en médecine clinique. Médecine thérapeutique. 2003;9:93-100.

22. Deccache A, Meremans P. L'éducation pour la santé des patients : au carrefour de la médecine et des sciences humaines. In : Sandrin-Berthon B. L'éducation du patient au secours de la médecine. Paris : P.U.F, $2000: 147-67$.

23. Cherbonnier A. Agir en promotion de la santé : un peu de méthode. Bruxelles: Question santé, 2000.

24. Schön D-A. Vers une nouvelle épistémologie de la profession face à la crise du savoir professionnel. In : Thomas A. et Ploman E.W. Savoir et développement permanent : une perspective mondiale. Toronto: OISE Press 1986:66-93.

25. Schön D-A. Le tournant réflexif. Pratiques éducatives et études de cas. Montréal : Logiques, 1996.

26. Perrenoud Ph. La place des sciences humaines et sociales dans l'expertise et les savoirs professionnels des enseignants. Rencontres du Réseau Éducation et Formation. Montréal: UQAM, 2001.

27. Vial M. L'auto-évaluation comme auto-questionnement. En questions 1997;12:143-98. 
28. Jonnaert P. Compétences et socio-constructivisme. Un cadre théorique. Bruxelles : De Boeck, 2002.

29. Falcy J-P. Ce qu'en dit Aristote. La problématique d'une discipline à l'autre. Paris : Adapt éditions 1997.

30. Meyer M. De la problématologie : philosophie, science et langage. Bruxelles : Mardaga, 1986.

31. Legrand M. Mathématiques, mythe ou réalité, un point de vue éthique sur l'enseignement scientifique. In : J.P. Falcy La problématique d'une discipline à l'autre, Paris : Adapt, 1997:59-88.

32. Hébrard P. Initiation à la recherche sur les pratiques et formation professionnelle : quels dispositifs pour apprendre à penser sa pratique? Recherche et formation 1994;17:23-34

33. Vial M. Se former pour évaluer. Se donner une problématique et élaborer des concepts. Bruxelles : De Boeck, 2001.

34. Eymard C. (Dir.) Le travail de fin d'études. S'initier à la recherche en soins et santé. Rueil-Malmaison : Lamarre, 2004.

35. Genthon M. Apprentissage-évaluation-recherche. Genèse des interactions complexes comme ouvertures régulatrices. En questions, 1997.

36. Ardoino J, Berger G. Du discours et des faits scientifiques dans les dites sciences de l'éducation. In : L'Année de la recherche en Sciences de l'éducation. Paris : PUF, 1997:5-55.

37. Affergan F. La pluralité des mondes. Paris : Albin Michel, 1997.

38. Morin E. Les sept savoirs nécessaires à l'éducation du futur. Paris: Organisation des Nations Unies (Pour l'éducation, la science et la culture), 1999.
39. Eymard C. De la formation par la recherche aux caractéristiques d'une relation éducative en santé. Synthèse des travaux de recherche présentée en vue de l'habilitation à diriger les recherches en sciences de l'éducation. Aix en Provence : Université AixMarseille I, 2005.

40. Thines $G$, Lempereur A. Dictionnaire général des sciences humaines. Belgique : Louvain-La-Neuve, 1984.

41. Devereux G. De l'angoisse à la méthode dans les sciences $d u$ comportement. Paris : Flammarion (1967), 1980.

42. Richelle M. Eloge des variations. In : Lautrey J. Universel et différentiel en psychologie. Paris : PUF, 1995:35-48.

43. Eymard $C$. Initiation à la recherche en soins et santé. Rueil-Malmaison : Lamarre, 2003.

44. Vermersh P. Prendre en compte la phénoménalité. Propositions pour une psychophénoménologie. Intelligence artificielle, 2005;19:57-75.

45. Cifali $M$. Le lien éducatif: contre-jour psychanalytique. Paris : PUF (1994) 1998.

46. GarfinKel H. Studies in Ethnomethodology. Englewood cliffs : Prentice Hall, 1967.

47. Morin E. Autonomie ou dépendance de la science. Quadrature Magazine de mathématiques pures et appliquées, 1997;30:5-11.

48. Develay M. A propos de l'éducation du patient. L'éducation du patient au secours de la médecine. Vendôme : PUF, 2000:186-98.

49. Favre D. Des neurosciences aux sciences de l'éducation : contribution à une épistémologie de la variance. Thèse de doctorat en psychologie et sciences de l'éducation. Lyon : université de Lyon 2, 1997.

50. Ricoeur P. Parcours de la reconnaissance. SaintAmand-Montrond: Stock, 2004.

Manuscrit reçu le 3 mars 2006; commentaires éditoriaux formulés le 23 mai 2006; accepté pour publication le 30 juin 2006. 